\title{
Compléments et corrections à la faune des Ephéméroptères d'Afrique du Nord. 1. Le genre Choroterpes Eaton, sensu stricto (Ephemeroptera)
}

\author{
A.G.B. Thomas 1 \\ B. Vitte ${ }^{2}$
}

Mots clés : Ephemeroptera, Choroterpes s. s., Afrique du Nord.

Description de l'imago o de Choroterpes (Ch.) volubilis n. sp. du Moyen Atlas et du Rif (Maroc), montrant les différences morphologiques des genitalia avec ceux de Ch. (Ch.) picteti (Eaton) et de $C h$. (Ch.) atlas Soldán et Thomas. Comparaison des répartitions de $C h$. volubilis et de $C h$. atlas en Afrique du Nord.

Additions and corrections to the Ephemeroptera fauna of North Africa. 1. The genus Choroterpes Eaton s.s.

Keywords: Ephemeroptera, Choroterpes s.s., North Africa.

A description of the male inago of Choroterpes (Ch.) volubilis n. sp. from the Middle Atlas and Rif (Marocco) showed morphological differences of the genitalia compared with those of Ch. (Ch.) picteri (Eaton) and Ch. (Ch.) atlas Soldán \& Thomas. The distribution of $\mathrm{Ch}$. volubilis is compared with that of $\mathrm{Ch}$. atlas in North Africa.

\section{Introduction}

Deux espèces du genre Choroterpes Eaton, 1881 sensu stricto sont actuellement connues d'Europe occidentale et des pays circum-méditerranéens de la moitié ouest de ce bassin : Ch. (Ch.) picteti (Eaton, 1871) européenne - terra typica : les environs de Genève - et Ch. (Ch.) atlas Soldán et Thomas, 1983 nord-africaine.

En effet, Ch. (Ch.) lindrothi Peters, 1980, décrite du sud du Maroc, appartient en réalité au sous-genre Euth raulus (voir Vitte et Thomas 1988). D'autre part, la synonymie $C h$. lusitanica Eaton, $1881=C h$. picteti - selon Eaton (1884), repris par Kimmins (1960) - nous paraît seulement probable et devra être vérifiée sur du matériel du Portugal.

1. Laboratoire d'Hydrobiologie, UA 695 du C.N.R.S., Universi té Paul Sabatier, 118 route de Narbonne, 31062 Touluuse Cédex, France.

2. Laboratoire de Faunistique, Département de Biologie, Facul té des Sciences, Université S Med Ben Abdallah, B.P. 1796 Atlas, Fes, Maroc.
Au cours de prospections de cours d'eau du Moyen Atlas et du Rif (Maroc), l'un de nous (B.V.) a récolté des larves dont l'élevage a révélé l'appartenance à une troisième espèce, nouvelle, dont voici la description.

\section{Choroterpes (Ch.) volubilis n. sp.}

Derivatio nominis : de Volubilis, ancienne ville romaine, située à proximité de nos stations de récolte.

Diagnose sommaire : comparativement à $C h$. $(C h$.) picteti et atlas, l'espèce est de grande taille, très foncée : les larves possèdent des branchies au système trachéen très ramifié.

Imago ơ (matériel conservé en alcool à $70^{\circ}$ ).

Tête.

Face brun moyen à carène plus claire. Vertex brun très foncé.

Antennes brun soutenu sauf le pédicelle, grisâtre vers l'apex. 
Ocelles gris clair, non cerclés, l'antérieur nettement plus petit.

Yeux : parties supérieures droite et gauche brun. rouge, net tement séparées sur toute leur longueur ; parties inférieures gris noirâtre.

Thorax.

Prothorax. Tergite et surtout sternite brun foncé. Une tache violacé noirâtre étendue sur la membrane articulaire entre coxa et bord latéral inférieur du tergite.

Mesothorax. Scutum et scutellum brun noirâtre à peu près uni et brillant. Mesosternum brun assez foncé brillant.

Metathorax plus clair et plus terne que le mesothorax.

Toutes les sutures latérales du thorax sont très claires, de couleur chair, et apparaissent donc très contrastées.

Pattes presque entièrement brun moyen - brun jaunâtre clair après la mue - sauf les coxas, la région apicale des fémurs et la base des tibias, brun foncé. Les fémurs portent d'autre part, sur les deux faces, une grande tache brun noirâtre médiane (fig. 1).

Ailes antérieures à membrane hyaline, sauf les champs $\mathrm{C}-\mathrm{Sc}$ et $\mathrm{Sc}-\mathrm{R} 1$ jaunâtres à bistres, excepté le tiers apical, plutôt laiteux. Aire ptérostigmatique portant une douzaine de nervures transverses peu ou pas ramifiées. Base de l'aile brun soutenu jusqu'au pont costal. Nervation brun jaunâtre. MA : rapport de la longueur de la tige à la longueur totale jusqu'au bord de l'aile, mesurée sur l'intercalaire entre MA1 et MA2, très voisin de 0,5 (compris entre 0,49 et 0,52 sur les 6 mesures effectuées). Liséré brun grisâtre net et tout à fait marginal sur le bord postérieur de l'aile.

Ailes postérieures en majeure partie hyalines - sauf la base, brune - à nervation brun grisâtre pour $\mathrm{C}$ et Sc, très claire ensuite. Projection costale peu marquée, à contour subsymétrique et d'un angle de $120^{\circ}$ environ (fig. 2).

\section{Abdomen.}

It est plus clair et plus terne que le thorax. Tergites brun foncé avec chacun, dans la région médiodorsale, une tache triangulaire claire - dirigée l'apex vers l'arrière - du $1^{\mathrm{cr}}$ au $9^{\mathrm{c}}$ segments, mais surtout visible du $2^{\mathrm{e}}$ au $8^{\mathrm{e}}$ inclus (fig. 3 ). Ces derniers portent aussi deux taches claires de chaque côté : l'une à l'angle antérieur du tergite, l'aut re à l'angle postérieur, de part et d'autre du spiracle. Le contour supérieur de ce dernier est surmonté d'une tache violacé noirâtre. $10^{\mathrm{e}}$ tergite brun noirâtre.

Sternites brun plus clair, jaunâtre assez uni ; ganglions nerveux maculés de pigment violacé.

Cerques brun moyen uni.

Genitalia.

Styligère brun moyen à dépression centrale peu marquée (fig. 4).

Styles brun grisâtre assez foncé sauf la région concave ventrale, amincie et de couleur chair. Encoche proximale exteme bien marquee. Contour proximal inteme de l'article 1 nettement, mais assez progressivement, convexe. Précisons toutefois que la courbure des styles n'étant pas plane et que la base de ces derniers étant oblique par rapport au plan sagittal, il est nécessaire de monter styligère et styles entre lame et lamelle (fig. 4) pour effectuer des comparaisons entre espèces. Dans l'en semble, si quelques différences peuvent être observées sur le contour proximal interne de l'article 1, elles sont difficilement généralisables en raison d'une importante variabilité individuelle.

Lobes péniens (fig. 5) longs, étroits, à contour interne rectiligne - en vue strictement ventrale et légèrement concaves extérieurement. Sous cette orientation, les trois espèces ne sont d'ailleurs pas très aisément distinguables. En vue latẻrale (fig. 6) au contraire, leur séparation est aisée. Les lobes péniens de $C h$. volu hilis (6v) présentent une courbure dorsale légèrement mais régulièrement convexe. De profil, leur extrémité ( $7 \mathrm{v}$ ) est en outre plus nettement élargie que chez Ch. atlas ( $6 \mathrm{a}$ et $7 \mathrm{a}$ ) chez laquelle la région distale est quelque peu sinueuse avec une faible concavité dorsale subterminale. Chez $\mathrm{Ch}$. picteri (6p et $7 p$ ) la région apicale (dépourvue d'épines sur nos exemplaires, contrairement aux deux autres espèces) est subaiguë et nettement recourbée ventralement.

Taille.

Longeurs en $\mathrm{mm}$ : aile antérieure $=8,1$ à $8,3 \mathrm{~mm}$; cerques $=8$ à $9,5 \mathrm{~mm}$. 


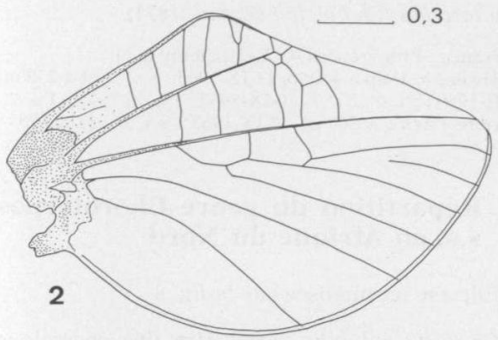

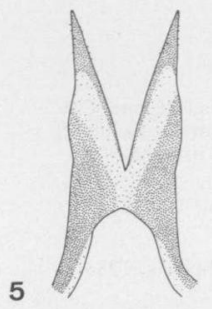

0.1
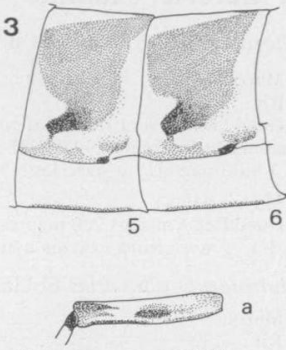

1 b
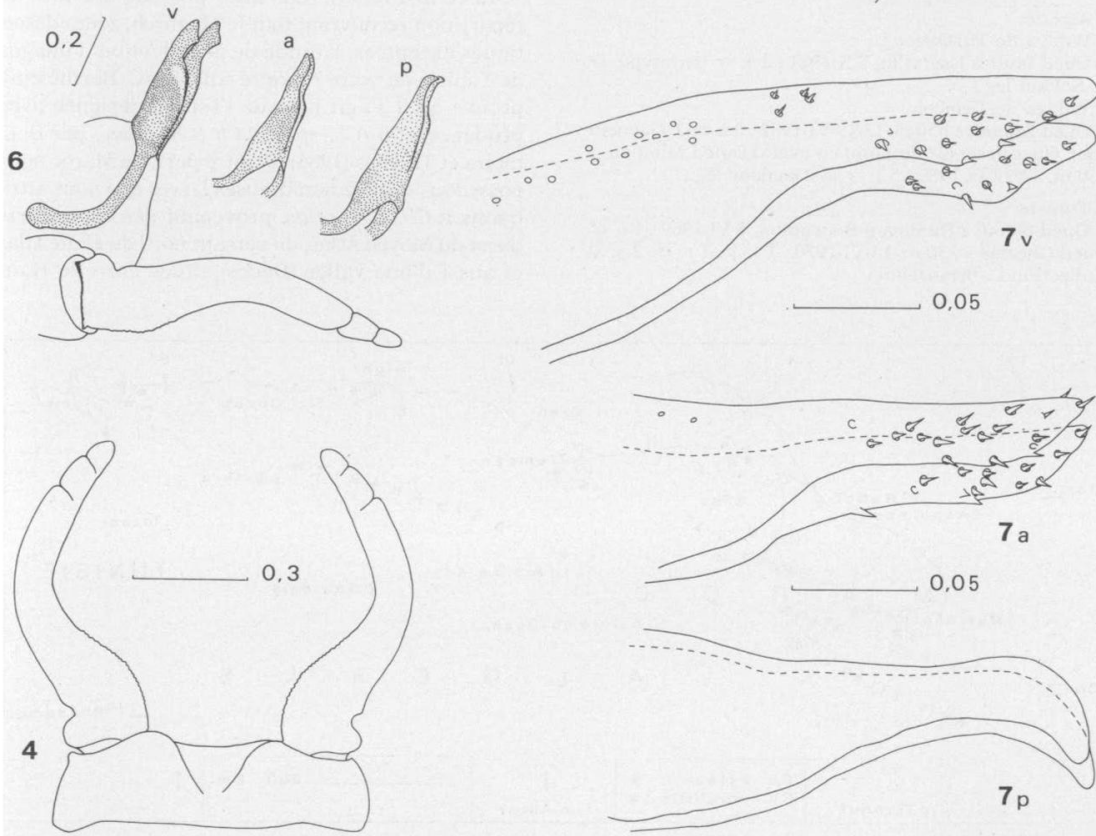

0.05

Fig. 1 à 3 : imago $\sigma$ de Choroterpes (Ch.) volubilis $n$. sp. Echelle en mm. ta et b : fémur antérieur, faces antéro-interne (a) et postéro-externe (b). 2 : aile postérieure. $3: 5^{\mathrm{c}}$ et $6^{\mathrm{c}}$ segments abdominaux vus de profil.

Fjg. 4 à 6 : genitalia or de Choroterpes sensu stricto. Echelle en mm. 4 : styligère et styles de $C h$. volubilis $n$. sp., aplatis ent re lame et lamelle. $5:$ pénis de $C h$. volubilis en vue ventrale. $6:$ pénis en vue latérale chez $C h$, volubilis (v), atlas (a) et picteti (p). 7 : détail de la région distale des lobes péniens chez ces mêmes espèces. 


\section{Matériel examiné}

Choroterpes (Ch.) volubilis $\mathrm{n}$. $\mathrm{sp}$.

Maroc.

Rif :

Oued Luukus vers $150 \mathrm{~m}$, au pont sur la $\mathrm{P} 28$ entre Chechaouen et Ouezzane, 14-IV-1987: 1 imago (i.) or (holotype) et 1 subimago (s.) \& avec leurs exuvies nymphales.

Moyen Atlas :

Oued Ben Çmin à $1260 \mathrm{~m}$, près d'Azrou, V.1985: 1 i. o et $1 \mathrm{~s}$. \& avec leurs exuvies nymphales.

Choroterpes (Ch.) atlas Soldán et Thomas, 1983.

Maroc.

Rif :

Oued Loukos à la même station, 14-IV-1987: 4 i. o et $1 \mathrm{~s}$. $\%$ avec leurs exuvies nymphales.

Algérie.

Wilaya de Tizi-Ouzou:

Oued Isser à Isserville, 5-X-1981: 1 i. o (paratype, Dr T. Soldán leg.).

Wilaya de Tlemcen :

Oued Khemis à $650 \mathrm{~m}, 13 / 19$.VI-1982:31. $\%$ (J. Gagneur leg.). Oued Isser ( $2^{c}$ du nom) en aval d'Ouled Mimoun, à $450 \mathrm{~m}, 14 / 22-\mathrm{IX}-198$ i : 5 i. $\sigma^{\circ}$ (J. Gagneur leg.).

Tunisie.

Oued Seloul à Hammam Bourguiba, 3-VI-1969: 1 i. $\sigma$. Oued Ghezala à $230 \mathrm{~m}, 13-\mathrm{VI}-1970: 1$ i. $\sigma, 1 \mathrm{~s} . \sigma, 2 \mathrm{~s}$. 8 (collection C. Berthélemy).
Choroterpes (Ch.) picteti (Eaton, 1871).

France : Prépyrénées (C. Berthélemy leg.).

Rivière le Volp à $460 \mathrm{~m}, 11-1 X-1963: 1 \mathrm{~s}$. O et à $240 \mathrm{~m}$, VIII-1959: 5 i. $\sigma, 2$ i. $Q, 10-1 X-1963: 1$ i. $\sigma, 7$ s. $\sigma, 1$ s. $Q$. Rivière l'Arize à $300 \mathrm{~m}, 13-\mathrm{IX}-1963: 9$ i. $\sigma, 21-\mathrm{X}-1965$ : 1 i. $\sigma$.

\section{Répartition du genre Choroterpes s.s. en Afrique du Nord}

Elle est schématisée sur la fig. 8.

Ch. (Ch.) volubilis paraît ètre une endémique marocaine.

Au contraire, $\mathrm{Ch}$. (Ch.) atlas possède une aire de répartition recouvrant tout le Maghreb, zones désertiques exceptées. L'étude de la collection d'imagos de Tunisie de notre regret té collègue $\mathrm{C}$. Berthélemy prouve qu'il s'agit bien de l'espèce désignée avec prudence " Ch. (Ch.) sp. = ? Ch. (Ch.) atlas " par Bou. maiza et Thomas (1986). D'autre part, du Maroc nous possédons d'assez nombreuses larves que nous attribuons à $C h$. (Ch.) atlas, provenant des contreforts ouest du Moyen Atlas, du versant nord du Haut Atlas et aussi d'une vallée (Dades) située entre le Haut

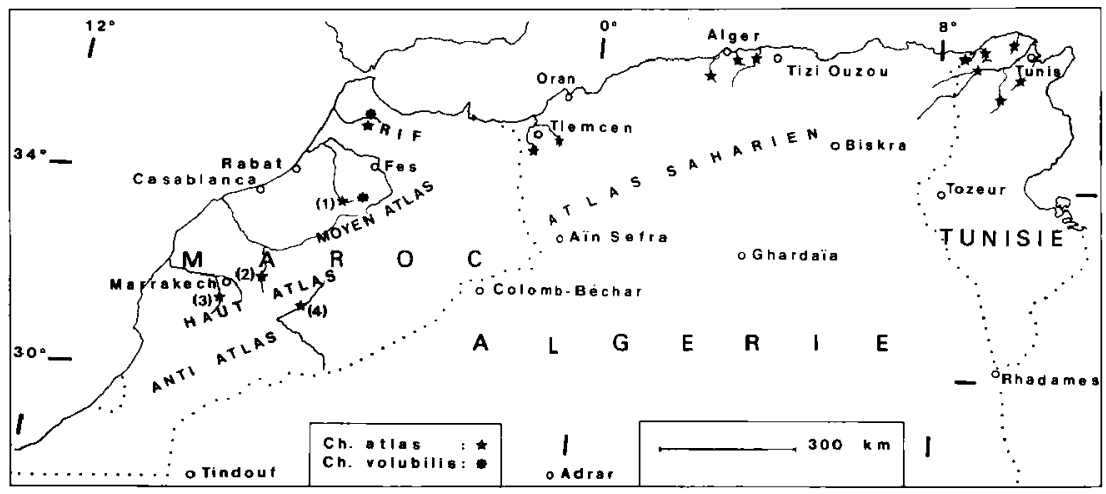

Fig. 8: répartition des espèces du genre Choroterpes sensu stricto en Afrique du Nord. (1) à (4) : matériel larvaire seulement (A. Thomas dét.). $1=0$. Beth (B. Vitte leg.) : $2=0$. Lakhdar (A. Bouzidi leg.); $3=0$. N'Fiss (A. Ajakane leg.) ; $4=0$. Dades (A. Bouzidi, J. Giudicelli et A. Thomas leg.).

N'est mentionnée qu'une seule station par cours d'eau principal. Données pour la Tunisie : Boumaiza et Thomas (1986) : pour l'Algérie : Soldán et Thomas (1983), Gagneur et Thomas (1988). 
Atlas et l'Anti Atlas. Cette dernière citation est, à notre connaissance, la plus méridionale pour cette espèce.

Les mentions de Ch. (Ch.) picteri d'Afrique du Nord (Lestage 1925, Dakki et El Agbani 1983, Kraiem 1986) doivent maintenant être considérées comme inexactes.

Nous remercions M.J. Gagneur pour avoir mis à notre disposition sa collection d'imagos de $C h$. (Ch.) atlas d'Algérie.

\section{Travaux citès}

Boumaiza (M.) et Thomas (A.G.B.). 1986. - Répartition et écologie des Ephéméroptères de Tunisie (1 ere partie) (Insecta, Ephemeropteral. Archs Inst. Pasteur Tunis, 63 (4) : 567-599.

Dakki (M.) et El Agbani (M.A.) 1983. - Ephéméroptères d'Af rique du Nord. 3. Elements pour la connaissance de la faume marocaine. Bull Inst scient. Rabat, $7: 115 \cdot 126$.

Eaton (A.E.). 1871. - A monograph on the Ephemeridae. Trans. ent. Soc. Lond, 1871 (1): $1-164+6 \mathrm{pl}$.

Eaton (A.E.). 1881. - An announcement of new genera of the Ephemeridac, Ent. mon. Mag., 17: 191-197.
Eaton (A.E.). 1883-88. - A revisional monograph of recent Ephe. meridae or mayflics. Trans. Linn. Soc. Land, Sec. Ser, Zool. $1-352+65 \mathrm{pl}$

Gagneur $(J$.) et Thomas (A.G.B.) 1988 _ Contribution à la connais. sance des Ephéméroptères d'Algérie. I. Répartition et écolo. gie (1 ere partie). Bull Soc Hist, nat. Toulouse, 124 : sous presse.

Kimmins (D.E.). 1960. - The Ephemeroptera types of species described by A.E. Eaton, R. Mc Lachlan and F. Walker. Bull. Br. Mus. (Nat Hist.), Entomology, 9 (4) : 269.318

Kraiem (M.). 1986. - Contribution à l'étude hydrobiologique de trois cours d'eau du Nord-Ouest de la Tunisie. Présentation, physicomchimie t aperçu faunistique. Bull. mens. Soc. Linr. Lyon 55 (3) : 96-104.

Lestage (J.A.). 1925. - Ephéméroptères, Plécoptères et Trichoptères recueillis en Algérie par M.H. Gaut hier et liste des especes connues actuellement de l"Afrique du Nord. Bull. Soc. Hist. nat. Afr. N., 16:8:18.

Peters (W.E.) 1980. - Choroterpes (Choroterpes) lindrothi, a new species of mayfly from Morocco (Ephemeroptera : Leptophlebiidae). Entomologia Gerteralis, $6(2-4): 371-373$.

Soldán (T.) et Thomas (A.G.B.). 1983. - New and little known species of mayflies (Ephemeroptera) from Algetia. Acta ent. bohe. moslov, 80: $356-376$

Vitte (B.) et Thomas (A.G.B.). 1988. - Compléments et corrections à la faune des Ephéméroptères d'Afrique du Nord. 2. Le genre Choroterpes Eaton, solis-genre Euthroulws Barnard. En préparation. 